\title{
Teaching Syntax by Adversarial Distraction
}

\author{
Juho Kim \\ University of Illinois \\ Urbana, IL \\ juhokim2@illinois.edu \\ Christopher Malon \\ NEC Laboratories America \\ Princeton, NJ \\ malon@nec-labs.com
}

\author{
Asim Kadav \\ NEC Laboratories America \\ Princeton, NJ \\ asimanec-labs.com
}

\begin{abstract}
Existing entailment datasets mainly pose problems which can be answered without attention to grammar or word order. Learning syntax requires comparing examples where different grammar and word order change the desired classification. We introduce several datasets based on synthetic transformations of natural entailment examples in SNLI or FEVER, to teach aspects of grammar and word order. We show that without retraining, popular entailment models are unaware that these syntactic differences change meaning. With retraining, some but not all popular entailment models can learn to compare the syntax properly.
\end{abstract}

\section{Introduction}

Natural language inference (NLI) is a task to identify the entailment relationship between a premise sentence and a hypothesis sentence. Given the premise, a hypothesis may be true (entailment), false (contradiction), or not clearly determined (neutral). NLI is an essential aspect of natural language understanding. The release of datasets with hundreds of thousands of example pairs, such as SNLI (Bowman et al., 2015) and MultiNLI (Williams et al., 2018), has enabled the development of models based on deep neural networks that have achieved near human level performance.

However, high accuracies on these datasets do not mean that the NLI problem is solved. Annotation artifacts make it possible to correctly guess the label for many hypotheses without even considering the premise (Gururangan et al., 2018; Poliak et al., 2018; Tsuchiya, 2018). Successful trained systems can be disturbed by small changes to the input (Glockner et al., 2018; Naik et al., 2018).

In this paper, we show that existing trained NLI systems are mostly unaware of the relation between syntax and semantics, particularly of how word order affects meaning. We develop a technique, "adversarial distraction," to teach networks to properly use this information. The adversarial distraction technique consists of creating pairs of examples where information matching the premise is present in the hypothesis in both cases, but differing syntactic structure leads to different entailment labels. We generate adversarial distractions automatically from SNLI and an NLI dataset derived from FEVER (Thorne et al., 2018), thus augmenting the datasets. We observe the behavior of several existing NLI models on the added examples, finding that they are mostly unaware that the syntactic changes have affected the meaning. We then retrain the models with the added examples, and find whether these weaknesses are limitations of the models or simply due to the lack of appropriate training data.

\section{Related work}

Datasets for NLI: SNLI and MultiNLI are both based on crowdsourced annotation. In SNLI all the premises came from image captions (Young et al., 2014), whereas MultiNLI collected premises from several genres including fiction, letters, telephone speech, and a government report. SciTail (Khot et al., 2018) constructed more complicated hypotheses based on multiple-choice science exams, whose premises were taken from web text. More recently, FEVER introduced a fact verification task, where claims are to be verified using all of Wikipedia. As FEVER established ground truth evidence for or against each claim, premises can be collected with a retrieval module and labeled as supporting, contradictory, or neutral for an NLI dataset.

Neural network based NLI systems: Dozens of neural network based models have been submitted to the SNLI leaderboard. Some systems have 
been developed based on sentence representations (Conneau et al., 2017; Nie and Bansal, 2017), but most common models apply attention between tokens in the premise and hypothesis. We focus on three influential models of this kind: Decomposable Attention (Parikh et al., 2016), ESIM (Chen et al., 2017), and a pre-trained transformer network (Radford et al., 2018) which obtains stateof-the-art results for various NLI datasets including SNLI and SciTail.

Adversarial examples for NLI systems: Jia and Liang (2017) introduced the notion of distraction for reading comprehension systems by trying to fool systems for SQuAD (Rajpurkar et al., 2016) with information nearly matching the question, added to the end of a supporting passage. Glockner et al. (2018) showed that many NLI systems were confused by hypotheses that were identical to the premise except for the replacement of a word by a synonym, hypernym, co-hyponym, or antonym. Naik et al. (2018) found that adding the same strings of words to NLI examples without changing the logical relation could significantly change results, because of word overlap, negation, or length mismatches.

Other work (Kang et al., 2018; Zhao et al., 2018) aimed to improve model robustness in the framework of generative adversarial networks (Goodfellow et al., 2014). Ribeiro et al. (2018) generated semantically equivalent examples using a set of paraphrase rules derived from a machine translation model. In contrast to these kinds of adversarial examples, we focus on the model not being sensitive enough to small changes that do change meaning.

\section{Teaching Syntax}

Our adversarial examples attack NLI systems from a new direction: not in their failure to capture the relations between words as in Glockner et al. (2018), but their failure to consider the syntax and word order in premises and hypotheses to decide upon the entailment relation. As position-agnostic approaches such as Decomposable Attention and SWEM (Shen et al., 2018) provide competitive baselines to existing datasets, the power of models to interpret token position information has not been rigorously tested.

\subsection{Passive voice}

We first evaluate and teach the use of the passive voice. By changing a hypothesis to passive, we can obtain a semantically equivalent hypothesis with identical tokens except for a different conjugation of the verb, the insertion of the word "by," and a different word order.

To perform the conversion, we use semantic role labeling results of SENNA (Collobert et al., 2011) to identify verbs and their ARG0 and ARG1 relations. We change the form of the verb and move the ARG0 and ARG1 phrases to opposite sides of the verb to form the passive. Here, we use head words identified in dependency parsing results and the part-of-speech tagging information of the verb from spaCy (Honnibal and Johnson, 2015) to change the verb form correctly according to the plurality of these nouns and the tense of the verb. The transformation is applied only at the root verb identified in dependency parsing output.

If the addition of the passive were the only augmentation, models would not be learning that word order matters. Thus, in the cases where the original pair is an entailment, we add an adversarial distraction where the label is contradiction, by reversing the subject and object in the hypothesis after transformation to passive. We call this the passive reversal. We filter out cases where the root verb in the hypothesis is a reciprocal verb, such as "meet" or "kiss," or a verb appearing with the preposition "with," so that the resulting sentence is surely not implied by the premise if the original is. For example, the hypothesis, "A woman is using a large umbrella" (entailment), generates the passive example, "A large umbrella is being used by a woman" (entailment), and the passive reversal, "A woman is being used by a large umbrella" (contradiction).

\subsection{Person reversal}

One weakness of adversarial distraction by passive reversal is that many hypotheses become ridiculous. A model leveraging language model information can guess that a hypothesis such as "A man is being worn by a hat" is a contradiction without considering the premise. Indeed, when we train a hypothesis only baseline (Poliak et al., 2018) with default parameters using the SNLI dataset augmented with passive and passive reversal examples, $95.98 \%$ of passive reversals are classified correctly from the hypothesis alone, while only 


\begin{tabular}{lcccccc}
\hline & \multicolumn{3}{c}{ SNLI } & \multicolumn{3}{c}{ FEVER } \\
& original & passive & passive rev & original & person rev & birthday \\
\hline \hline \# train & 549,367 & 129,832 & 39,482 & 602,240 & 3,154 & 143,053 \\
\# validation & 9,842 & 2,371 & 724 & 42,541 & 95 & 9,764 \\
\# test & 9,824 & 2,325 & 722 & 42,970 & 69 & 8,880 \\
\hline
\end{tabular}

Table 1: The number of examples in the original SNLI and FEVER data, and the number of examples generated for each adversarial distraction.

$67.89 \%$ of the original and $64.69 \%$ of the passive examples are guessed correctly.

To generate more plausible adversarial distractions, we try reversing named entities referring to people. Most person names should be equally likely with or without reversal, with respect to a language model, so the generated examples should rely on understanding the syntax of the premise correctly.

SNLI generally lacks named entities, because it is sourced from image captions, so we consider FEVER data instead. The baseline FEVER system (Thorne et al., 2018) retrieves up to five sentences of potential evidence from Wikipedia for each claim, by comparing TFIDF scores. We label each of these evidence/claim pairs as entailment or contradiction, according to the claim label, if the evidence appears in the ground truth evidence set, and as neutral otherwise. Because the potential evidence is pulled from the middle of an article, it may be taken out of context with coreference relations unresolved. To provide a bit of context, we prefix each premise with the title of the Wikipedia page it comes from, punctuated by brackets.

Our person reversal dataset generates contradictions from entailment pairs, by considering person named entities identified by SENNA in the hypothesis, and reversing them if they appear within the ARG0 and ARG1 phrases of the root verb. Again, we filter examples with reciprocal verbs and the preposition "with." For example, the FEVER claim, "Lois Lane's name was taken from Lola Lane's name" (entailment), leads to a person reversal of "Lola Lane's name was taken from Lois Lane's name" (contradiction).

To compare the plausibility of the examples, we train the same hypothesis only baseline on the FEVER dataset augmented with the person reversals. It achieves $15.94 \%$ accuracy on the added examples, showing that the person reversals are more plausible than the passive reversals.

\subsection{Life spans}

Our third adversarial distraction (birthday) involves distinguishing birth and death date information. It randomly inserts birth and death dates into a premise following a person named entity, in parentheses, using one of two date formats. If it chooses a future death date, no death date is inserted. A newly generated hypothesis randomly gives a statement about either birth or death, and either the year or the month, with a label equally balanced among entailment, contradiction, and neutral. For half of the contradictions it simply reverses the birth and death dates; otherwise it randomly chooses another date. For the neutral examples it asks about a different named entity, taken from the same sentence if possible.

For example, a birthday and death date are randomly generated to yield the premise, "[Daenerys Targaryen] Daenerys Targaryen is a fictional character in George R. R. Martin's A Song of Ice and Fire series of novels, as well as the television adaptation, Game of Thrones, where she is portrayed by Emilia Clarke (April 25, 860 - November 9, 920)," and hypothesis, "Emilia Clarke died in April" (contradiction).

\section{Evaluation}

\subsection{Experiments}

We consider three NLI systems based on deep neural networks: Decomposable Attention (DA) (Parikh et al., 2016), ESIM (Chen et al., 2017), and a Finetuned Transformer Language Model (FTLM) (Radford et al., 2018). For Decomposable Attention we take the AllenNLP implementation (Gardner et al., 2017) without ELMo features (Peters et al., 2018); for the others, we take the releases from the authors. We modify the code released for FTLM to support entailment classification, following the description in the paper.

For the FEVER-based datasets, for DA and ESIM, we reweight each class in the training data 


\begin{tabular}{lcccccc}
\hline & \multicolumn{3}{c}{ SNLI } & \multicolumn{3}{c}{ FEVER } \\
& Original & Passive & Passive rev. & Original & Person rev. & Birthday \\
\hline \hline DA & .8456 & .8301 & .0111 & $.8416(.1503)$ & .0435 & .2909 \\
ESIM & .8786 & .8077 & .0139 & $.8445(.3905)$ & .0290 & .3134 \\
FTLM & .8980 & .8430 & .0540 & $.9585(.6656)$ & .0000 & .2953 \\
\hline
\end{tabular}

Table 2: Accuracy and (Cohen's Kappa) when training on original SNLI or FEVER data and testing on original or added examples.

\begin{tabular}{lccccccc}
\hline & \multicolumn{3}{c}{ SNLI } & \multicolumn{2}{c}{ Person rev. } & \multicolumn{2}{c}{ Birthday } \\
& Original & Passive & Passive rev. & Original & Added & Original & Added \\
\hline \hline DA & .8517 & .7333 & .5042 & $.8552(.1478)$ & .1449 & $.8925(.1550)$ & .4700 \\
ESIM & .8781 & .8667 & .9833 & $.8406(.3809)$ & .6232 & $.8721(.4404)$ & .9684 \\
FTLM & .8953 & .8920 & .9917 & $.9581(.6610)$ & .7536 & $.9605(.6809)$ & .9926 \\
\hline
\end{tabular}

Table 3: Accuracy and (Cohen's Kappa) when training on augmented SNLI (SNLI + passive + passive reversal) or augmented FEVER (FEVER + person reversal or FEVER + birthday) and testing on original or added examples.

in inverse proportion to its number of examples. This reweighting is necessary to produce nontrivial (most frequent class) results; the NLI training set we derive from FEVER has $92 \%$ neutral, $6 \%$ entailment, and $2 \%$ contradiction examples. FTLM requires no such reweighting. When evaluating on the original FEVER examples, we report Cohen's Kappa between predicted and ground truth classifications, in addition to accuracy, because the imbalance pushes DA and ESIM below the accuracy of a trivial classifier.

Whereas FTLM uses a byte-pair encoding vocabulary (Sennrich et al., 2016) that can represent any word as a combination of subword tokens, DA and ESIM rely on word embeddings from GloVe (Pennington et al., 2014), with a single out-ofvocabulary (OOV) token shared for all unknown words. Therefore it is unreasonable to expect DA and ESIM not to confuse named entities in FEVER tasks. We extend each of these models by allocating 10,000 random vectors for out-ofvocabulary words, and taking a hash of each OOV word to select one of the vectors. The vectors are initialized from a normal distribution with mean 0 and standard deviation 1.

\subsection{Results}

When we train the three models using original SNLI without augmentation, the models have slightly lower performance on the passive examples than the original data. However, all three models fail to properly classify the passive reversal data: without training, it looks too similar to the original hypothesis. The augmented data succeeds in training two out of three of the models about the passive voice: ESIM and FTLM can classify the passive examples with approximately the accuracy of the original examples, and with even higher accuracy, they can pick out the passive reversals as preposterous. However, DA cannot do better than guess whether a passive sentence is reversed or not. This is because its model is defined so that its output is invariant to changes in word order. Because it must consider the possibility of a passive reversal, its performance on the passive examples actually goes down after training with augmentations.

Person reversal also stumps all three models before retraining. Of course DA can get a person reversal right only when it gets an original example wrong, because of its insensitivity to word order. ESIM and FTLM find person reversals to be more difficult than the original examples. Compared to passive reversal, the lack of language hints seems to make the problem more challenging. However, the multitude of conditions necessary to perform a person reversal makes the added examples less than $1 \%$ of the overall training data.

No model trained on FEVER initially does better than random guessing on the birthday problem. By training with augmented examples, ESIM and FTLM learn to use the structure of the premise properly to solve this problem. DA learns some hints after retraining, but essentially the problem depends on word order, which it is blind to. It is noteworthy that the performance of all three mod- 
els on the original data improves after the birthday examples are added, unlike the other two augmentations, where performance remains the same. Four percent of the original FEVER claims use the word "born" or "died," and extra practice with these concepts proves beneficial.

\section{Discussion}

We have taken two basic aspects of syntax, the equivalence of passive and active forms of the same sentence, and the distinction between subject and direct object, and shown that they are not naturally learned through existing NLI training sets. Two of the models we evaluated could master these concepts with added training data, but the Decomposable Attention model could not even after retraining.

We automatically generated training data to teach these syntactic concepts, but doing so required rather complicated programs to manipulate sentences based on parsing and SRL results. Generating large numbers of examples for more complicated or rarer aspects of syntax will be challenging. An obvious difficulty in extending our approach is the need to make a distraction template that affects the meaning in a known way. The other difficulty lies in making transformed examples plausible enough not to be rejected by language model likelihood.

\section{References}

Samuel Bowman, Gabor Angeli, Christopher Potts, and Christopher Manning. 2015. A large annotated corpus for learning natural language inference. In Proceedings of the Conference on Empirical Methods in Natural Language processing (EMNLP).

Qian Chen, Xiaodan Zhu, Zhen-Hua Ling, Si Wei, Hui Jiang, and Diana Inkpen. 2017. Enhanced LSTM for natural language inference. In Proceedings of the 55th Annual Meeting of the Association for Computational Linguistics (ACL) Volume 1, pages 1657 1668.

Ronan Collobert, Jason Weston, Léon Bottou, Michael Karlen, Koray Kavukcuoglu, and Pavel Kuksa. 2011. Natural language processing (almost) from scratch. Journal of Machine Learning Research (JMLR), 12:2493-2537.

Alexis Conneau, Douwe Kiela, Holger Schwenk, Loïc Barrault, and Antoine Bordes. 2017. Supervised learning of universal sentence representations from natural language inference data. In Proceedings of the Conference on Empirical Methods in Natural Language Processing (EMNLP), pages 670-680.
Matt Gardner, Joel Grus, Mark Neumann, Oyvind Tafjord, Pradeep Dasigi, Nelson F. Liu, Matthew Peters, Michael Schmitz, and Luke S. Zettlemoyer. 2017. AllenNLP: A deep semantic natural language processing platform. CoRR, abs/1803.07640.

Max Glockner, Vered Shwartz, and Yoav Goldberg. 2018. Breaking NLI systems with sentences that require simple lexical inferences. In Proceedings of the 56th Annual Meeting of the Association for Computational Linguistics (ACL) Volume 2, pages 650655 .

Ian Goodfellow, Jean Pouget-Abadie, Mehdi Mirza, Bing $\mathrm{Xu}$, David Warde-Farley, Sherjil Ozair, Aaron Courville, and Yoshua Bengio. 2014. Generative adversarial nets. In Advances in Neural Information Processing Systems (NIPS) 27, pages 2672-2680.

Suchin Gururangan, Swabha Swayamdipta, Omer Levy, Roy Schwartz, Samuel Bowman, and Noah A. Smith. 2018. Annotation artifacts in natural language inference data. In Proceedings of the 2018 Conference of the North American Chapter of the Association for Computational Linguistics: Human Language Technologies (NAACL-HLT) Volume 2, pages 107-112.

Matthew Honnibal and Mark Johnson. 2015. An improved non-monotonic transition system for dependency parsing. In Proceedings of the Conference on Empirical Methods in Natural Language Processing (EMNLP), pages 1373-1378.

Robin Jia and Percy Liang. 2017. Adversarial examples for evaluating reading comprehension systems. In Proceedings of the Conference on Empirical Methods in Natural Language Processing (EMNLP), pages 2021-2031.

Dongyeop Kang, Tushar Khot, Ashish Sabharwal, and Eduard Hovy. 2018. Adversarial training for textual entailment with knowledge-guided examples. In Proceedings of the 56th Annual Meeting of the Association for Computational Linguistics (ACL).

Tushar Khot, Ashish Sabharwal, and Peter Clark. 2018. SciTail: A textual entailment dataset from science question answering. In Proceedings of the ThirtySecond AAAI Conference on Artificial Intelligence.

Aakanksha Naik, Abhilasha Ravichander, Norman Sadeh, Carolyn Rose, and Graham Neubig. 2018. Stress test evaluation for natural language inference. In Proceedings of the 27th International Conference on Computational Linguistics (COLING), Santa Fe, New Mexico, USA.

Yixin Nie and Mohit Bansal. 2017. Shortcut-stacked sentence encoders for multi-domain inference. In Proceedings of the 2nd Workshop on Evaluating Vector Space Representations for NLP, RepEval@EMNLP, pages 41-45. 
Ankur Parikh, Oscar Täckström, Dipanjan Das, and Jakob Uszkoreit. 2016. A decomposable attention model for natural language inference. In Proceedings of the Conference on Empirical Methods in Natural Language Processing (EMNLP), pages 22492255 .

Jeffrey Pennington, Richard Socher, and Christopher D. Manning. 2014. GloVe: Global vectors for word representation. In Proceedings of the Conference on Empirical Methods in Natural Language Processing (EMNLP), pages 1532-1543.

Matthew E. Peters, Mark Neumann, Mohit Iyyer, Matt Gardner, Christopher Clark, Kenton Lee, and Luke Zettlemoyer. 2018. Deep contextualized word representations. In Proceedings of the Conference of the North American Chapter of the Association for Computational Linguistics: Human Language Technologies (NAACL-HLT) Volume 1, pages 22272237.

Adam Poliak, Jason Naradowsky, Aparajita Haldar, Rachel Rudinger, and Benjamin Van Durme. 2018. Hypothesis only baselines in natural language inference. In Proceedings of the Joint Conference on Lexical and Computational Semantics (*Sem).

Alec Radford, Karthik Narasimhan, Tim Salimans, and Ilya Sutskever. 2018. Improving language understanding by generative pre-training. In OpenAI Blog.

Pranav Rajpurkar, Jian Zhang, Konstantin Lopyrev, and Percy Liang. 2016. SQuAD: 100, 000+ questions for machine comprehension of text. In Proceedings of the Conference on Empirical Methods in Natural Language Processing (EMNLP), pages 2383-2392.

Marco Tulio Ribeiro, Sameer Singh, and Carlos Guestrin. 2018. Semantically equivalent adversarial rules for debugging NLP models. In Proceedings of the 56th Annual Meeting of the Association for Computational Linguistics (ACL) Volume 1, pages 856-865.

Rico Sennrich, Barry Haddow, and Alexandra Birch. 2016. Neural machine translation of rare words with subword units. In Proceedings of the 54th Annual Meeting of the Association for Computational Linguistics (ACL) Volume 1, pages 1715-1725.

Dinghan Shen, Guoyin Wang, Wenlin Wang, Martin Renqiang Min, Qinliang Su, Yizhe Zhang, Chunyuan Li, Ricardo Henao, and Lawrence Carin. 2018. Baseline needs more love: On simple wordembedding-based models and associated pooling mechanisms. In Proceedings of the 56th Annual Meeting of the Association for Computational Linguistics (ACL) Volume 1, pages 440-450.

James Thorne, Andreas Vlachos, Christos Christodoulopoulos, and Arpit Mittal. 2018. FEVER: a large-scale dataset for fact extraction and verification. In Proceedings of the Conference of the North American Chapter of the Association for Computational Linguistics: Human Language Technologies (NAACL-HLT) Volume 1.

Masatoshi Tsuchiya. 2018. Performance impact caused by hidden bias of training data for recognizing textual entailment. In Proceedings of the Eleventh International Conference on Language Resources and Evaluation (LREC).

Adina Williams, Nikita Nangia, and Samuel Bowman. 2018. A broad-coverage challenge corpus for sentence understanding through inference. In Proceedings of the Conference of the North American Chapter of the Association for Computational Linguistics: Human Language Technologies (NAACL-HLT) Volume 1, pages 1112-1122.

Peter Young, Alice Lai, Micah Hodosh, and Julia Hockenmaier. 2014. From image descriptions to visual denotations: New similarity metrics for semantic inference over event descriptions. In Transactions of the Association for Computational Linguistics (TACL), volume 2, pages 67-78.

Zhengli Zhao, Dheeru Dua, and Sameer Singh. 2018. Generating natural adversarial examples. In Proceedings of the International Conference on Learning Representations (ICLR). 